\title{
Insertion of Nanoparticle Clusters into Vesicle Bilayers
}

\author{
Cécile Bonnaud, ${ }^{t, \pm,{ }^{\circ}}$ Christophe A. Monnier, ${ }^{+,{ }^{\circ}}$ Davide Demurtas, ${ }^{\S}$ Corinne Jud, ${ }^{\dagger}$ Dimitri Vanhecke, ${ }^{\dagger}$ \\ Xavier Montet, ${ }^{\perp}$ Ruud Hovius," Marco Lattuada, ${ }^{\dagger}$ Barbara Rothen-Rutishauser, ${ }^{\dagger, \|}$ and Alke Petri-Fink ${ }^{\dagger, \neq, *}$ \\ ${ }^{\dagger}$ Adolphe Merkle Institute, University of Fribourg, Route de I'Ancienne Papéterie CP209, 1723 Marly 1, Switzerland, ${ }^{\ddagger}$ Chemistry Department, University of Fribourg, \\ Chemin du Musée 9, 1700 Fribourg, Switzerland, IInterdisciplinary Center for Electron Microscopy, Ecole Polytechnique Fédérale de Lausanne, Station 15, \\ 1015 Lausanne, Switzerland, ${ }^{\perp}$ Radiology Department, Geneva University Hospital, Rue Gabrielle-Perret-Gentil 4, 1211 Genève 14, Switzerland, "Laboratory of \\ Physical Chemistry of Polymers and Membranes, Ecole Polytechnique Fédérale de Lausanne, Station 6, 1015 Lausanne, Switzerland, and "Respiratory Medicine, \\ Bern University Hospital, Inselspital, Freiburgstrasse, 3010 Bern, Switzerland. "These authors contributed equally to this work.
}

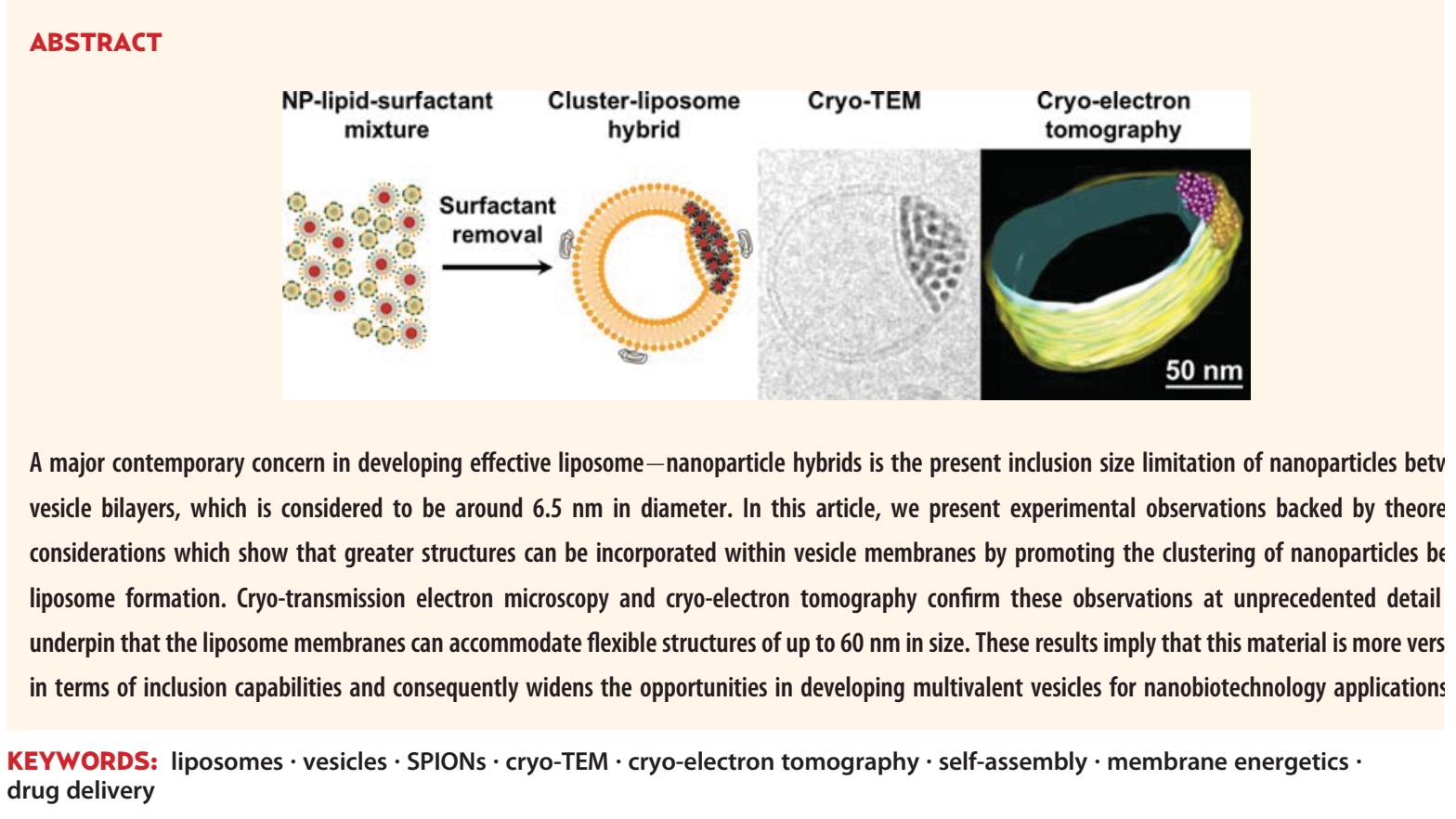
drug delivery

ombining nanoparticles with liposomes is a highly elaborative methodology in the emerging fields of nanomedicine and nanobiotechnology. Not only can the well-known benefits ${ }^{1}$ of liposomes be enhanced for specific applications, but their established scope of functionality (e.g., targeted drug delivery ${ }^{2-7}$ ) can also be widely expanded to fulfill additional tasks and thereby act as eclectic toolboxes at a nanoscale. ${ }^{8}$

Lipid bilayer membranes alone are significantly relevant materials for a broad range of academic disciplines. Their interactions with nanoparticles have been gradually studied over the past years, particularly in the context of nanoparticle transport across celIular membranes..$^{9-12}$ Notable experimental and theoretical studies have addressed different variables (e.g., the nanoparticle size or lipid composition) and their effects on adhesion and wrapping or nanoparticle-induced pore formation. ${ }^{12-15}$ These concepts have been complemented by, for example, molecular dynamics simulations ${ }^{16}$ or mesoscale thermodynamic modeling ${ }^{13}$ and provide, among others, more accurate descriptions of phospholipid membranes to determine the role and effects of nanoparticles on lipid bilayers in general.

In the context of stimuli-responsive liposome-nanoparticle hybrids for drug delivery, embedding hydrophobic nanoparticles directly within the liposomal lipid bilayer has become a popular approach. There are several standards in the literature on this subject, ${ }^{17-21}$ which also present operative methodologies to accomplish this task. In this wide-ranging field, leading studies have focused on using very small nanoparticles
* Address correspondence to alke.fink@unifr.ch. 
( $5 \mathrm{~nm}$ in diameter or below), ${ }^{8}$ as the curvature of monolayer lipids covered around small nanoparticles is too high, consequently promoting vesicle formation. ${ }^{18,20,22}$ On the other hand, spheres larger than approximately $6.5 \mathrm{~nm}$ in diameter, ${ }^{22}$ or around the overall bilayer thickness in general, ${ }^{13,23}$ rather induce lipid monolayer adsorption onto the surface and result in stable micelle-like structures. These norms have been repetitively validated in experimental studies, ${ }^{18,19}$ and larger nanoparticles (i.e., $>22 \mathrm{~nm}$ ) in turn are known to be wrapped up by a lipid bilayer. ${ }^{12,15}$

These boundaries can be cumbersome when designing application-oriented nanocontainers, as specific nanoparticle properties (i.e., optical, thermal and/or magnetic)—which make them desirable in the first place-are mostly governed by their size. Superparamagnetic iron oxide nanoparticles (SPIONs) are a general paradigm: their superparamagnetic features make them ideal thermoregulators ${ }^{24}$ for various medically oriented tasks (i.e., hyperthermia ${ }^{25,26}$ or drug release ${ }^{17,18,27,28}$ ) and are presently valued as contrast agents in magnetic resonance imaging. ${ }^{29-31}$ However, the size of the nanoparticles defines their effectiveness: small SPIONs are suboptimal for the aforementioned tasks, as their specific absorption rate at the relevant magnetic field frequencies and gradients is lower than larger ones. ${ }^{25}$ This momentarily presents a dilemma, as one has to choose between a stable embedding within the lipid bilayer and an optimally tuned material.

In this article, we demonstrate that the present application constraints can be circumvented by tuning the self-assembly steps of vesicles by surfactant dialysis. ${ }^{20,32}$ We pursued an abstract fundamental approach to push forward the co-assemblage of nanoparticles and lipid micelles to promote the incorporation of large clusters-consisting of hundreds of nanoparticles-within the resulting liposome bilayer. By endorsing the clustering of hydrophobic nanoparticles (in this instance, SPIONs) before liposome formation, the assembling vesicles were encouraged to grow and close in around these large structures.

Another noteworthy challenge in the field is the in-depth characterization of vesicles and the spatial location of nanoparticles within them. Favored highresolution imaging systems provide merely a projection of the sample, not the three-dimensional ultrastructure, and operate in a high vacuum environment. As a consequence, the question remains perplexing to answer whether the nanoparticles are where they are believed to be. In our study, we showed under unadulterated conditions that the clustered SPIONs were distinctly trapped between two lipid sheets by using cryo-transmission electron microscopy (cryo-TEM). Moreover, auxiliary three-dimensional analysis by cryoelectron tomography displayed the architecture and spatial arrangement of these lenticular-formed intrusions. These observations were finally underpinned by supporting theoretical considerations which emphasize that not only the size of the inclusive structure is determinant, as previously supposed, but also the shape as well.

\section{RESULTS}

As nanoparticles feature particular behaviors, especially when colloidal stability and aggregation are dictated by surfactants and phospholipids, ${ }^{33}$ an indepth understanding and analysis of all involved materials is indispensable. In this study, we used SPIONs coated with a hydrophobic layer of oleic acid (referred to as OA-SPIONs, Figure $1 \mathrm{~A}) .^{34}$ These nanoparticles were stabilized in an aqueous buffer with $\beta$-octyl glucoside (OG) acting as a surfactant ${ }^{35}$ (referred to as OG-OA-SPIONs) and preserving them in a metastable state. ${ }^{33}$ OG, a neutral charged detergent, was chosen due to its nonionic properties and its known ability to solubilize, purify, and crystallize membrane proteins without denaturation. ${ }^{36,37}$ The surfactant concentration was primarily set high above its specific critical micelle concentration (CMC, approximately $25 \mathrm{mM}^{38}$ ) at $130 \mathrm{mM}$ to ensure that the hydrophobic nanoparticles were entirely engulfed by a protective micelle (Figure 1B). SPIONs in both states were then investigated and compared by transmission electron microscopy (TEM) and cryo-TEM (Figure 1A-C) and displayed core diameters of $5.7 \pm 1.0 \mathrm{~nm}$ (mean \pm $S D$, Figure $1 A, B)$. Nevertheless, the individual nanoparticles assembled into spherical clusters once the protective surfactant shell was partially reduced by dialysis (Figure 1C), while still remaining above the CMC. This behavior was particularly relevant and desired for upcoming incorporation experiments and further confirmed by small-angle X-ray scattering (SAXS, Figure 1D). SPIONs covered by surfactant exhibited a diameter of $6.30 \pm 0.09 \mathrm{~nm}$ along with an increased forward scattering at a low scattering vector (Figure 1D), which is indicative for attractive forces between the nanoparticles. These interactions are highly dependent on the selected surfactant and consequently critical in this assembly.

In contrast to hydrophobic SPION stability in aqueous buffer, liposome integrity is compromised by high surfactant concentrations, ${ }^{32,39-41}$ which force the vesicles to undergo a transition from an organized structure to individual micelles (Suppl. Figure 1). However, removing the surfactant from this mixed solution (e.g., by dialysis) causes the liposomes to reassemble. ${ }^{32}$ On the basis of this circumstance, we added preformed liposomes to a solution of OG-OA-SPIONs (Figure 1E, left). The lipid composition of these vesicles was chosen in regard to future biomedical application and with a transition phase temperature around $42{ }^{\circ} \mathrm{C}$. PEGylated lipids were added to the formulation to equip the liposomes with stealth properties against the reticuloendothelial system and to allow for 

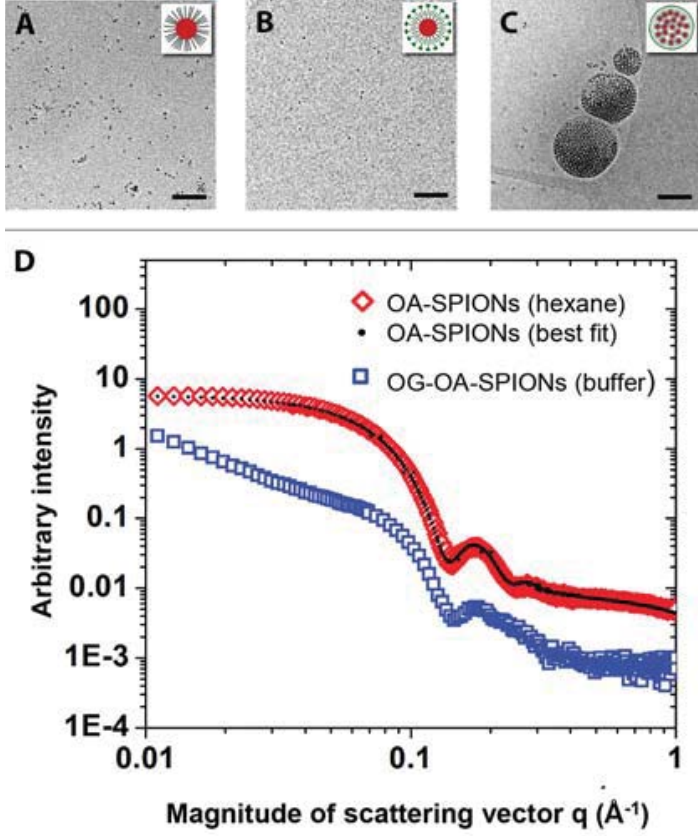

E

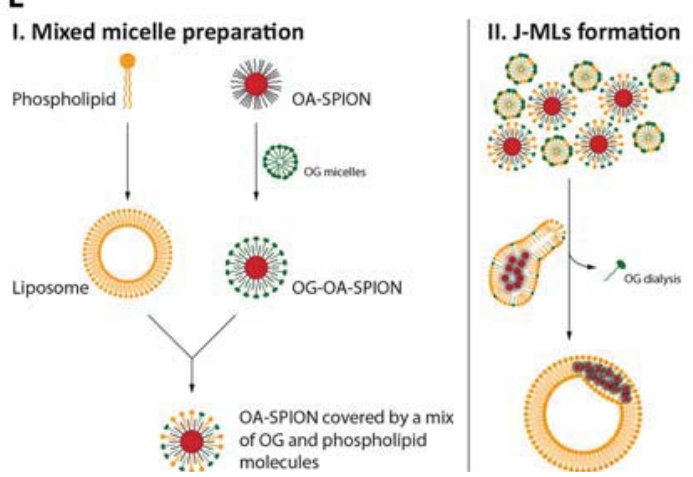

Figure 1. Hydrophobic nanoparticles were investigated before and after phase transfer. After nanoparticle synthesis, OA-SPIONs were dispersed in hexane for storage and characterized by TEM (A). The nanoparticles were then transferred to buffer by addition of $\beta$-octyl glucoside (OG) and characterized by cryo-TEM (B,C). The concentration of $\beta$-octyl glucoside was set far above the surfactant critical micelle concentration (CMC) to obtain individually dispersed OG-OA-SPIONs (B). After a partial surfactant removal, the nanoparticles assembled into spherical clusters (C). SAXS scattering curves before and after phase transfer (D) show that the individual particle sizes remained unchanged as demonstrated by the peak position. Scale bars = $100 \mathrm{~nm}$. The subsequent synthetic approach to obtain cluster-liposome hybrids is summarized in (E).

subsequent functionalization possibilities (e.g., with antibodies for specific targeting). ${ }^{1}$ The surfactant within the buffer disintegrated the vesicles, resulting in a diversified micellar suspension of surfactant, nanoparticles, and phospholipids. Following this, OG was slowly depleted by dialysis (Figure $1 \mathrm{E}$, right). The recreated vesicles (Figure $2 \mathrm{~A}$ ) were thoroughly analyzed by cryo-TEM in order to preserve the fragile structures and to prevent drying artifacts. Overlapping nanoparticles, mainly organized as clusters, and liposomes were immediately distinguishable. Most strikingly, the general majority had a Janus-like appearance, with all incorporated clusters diverged at one pole of the vesicle. High-magnification micrographs (Figure $2 \mathrm{~B}, \mathrm{C}$ ) showed a distinct bilayer unzipping around the clusters, hence demonstrating a major inclusion between the lipid sheets. Subsequent numerical analysis of the images revealed that more than $90 \%$ of all liposomes were uniformly encumbered with a reasonably sized nanoparticle cluster. The remaining structures exhibited either a sheet-like nanoparticle distribution within the membrane or were unloaded giant unilamellar vesicles. Stage tilting $\left( \pm 15^{\circ}\right.$, Suppl. Figure 2$)$ confirmed that both liposomes and clusters were associated with each other, and not coincidentally superimposed. ${ }^{42}$ Moreover, cryo-EM tomograms were generated to further study the architectural features in three dimensions (Figure 2D,E). Within the clusters, the individual nanoparticles were densely packed (Figure $2 \mathrm{E}$ ) with spacing distances around $1.5 \mathrm{~nm}$. An overall approximation of their inclusive sizes and morphologies could consequently be elaborated and showed that hundreds of nanoparticles were stacked between the lipid bilayer and hexagonally organized (Suppl. Figure 3 and suppl. movie). Moreover, the intrusion volume between the lipid sheets was measured to be approximately $128960 \mathrm{~nm}^{3}$, which corresponds to an estimated 660 nanoparticle-sized cluster (Figure 2E).

The reassembled cluster-liposome hybrids, termed Janus magnetic liposomes (J-MLs), were remarkably homogeneous, with an overall diameter around $150 \mathrm{~nm}$ (Suppl. Figure 4). Supplementary size measurements by dynamic light scattering prior to and after dialysis (Suppl. Figure 4A) supported the impression that the overall size distribution between the liposomes was analogous. Interestingly, these measurements highlighted that the reassembled vesicles exhibited a nearly identical radius compared to their preformed counterparts, with only a minor increase in distribution broadness. These observations were consistent with low-magnification cryo-TEM overview images (Suppl. Figure 4B,C).

To address the importance of beginning from individual micelles in this assembly process, we investigated the effect that varying initial surfactant concentrations has on the overall experimental efficiency (Figure 3). Three different initial surfactant (OG) concentrations with respect to the CMC (below, equal, and above) were correlated to cryo-TEM micrographs recorded after surfactant dialysis (Figure $3 \mathrm{~A}-\mathrm{C}$ ). At sublytic surfactant concentrations ( $<C M C$, Figure $3 A$ ), liposomal stability was not entirely compromised, yet the elasticity of the liposome membrane was affected. ${ }^{39,41}$ Polydisperse giant unilamellar liposomes were the outcome, most likely a result of induced fusion between the vesicles. Most nanoparticles irregularly aggregated into rigid sheets outside of the vesicles during the process (Figure $3 \mathrm{~A}$ ). An initial 

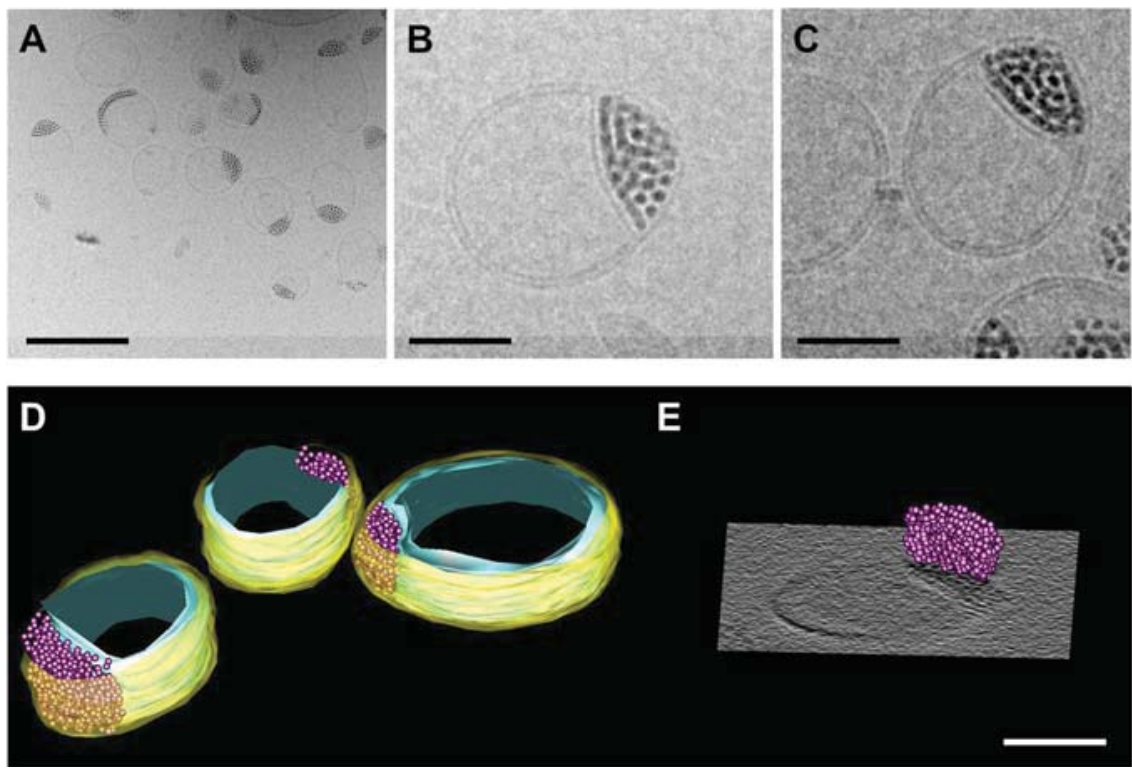

Figure 2. Reassembled cluster-liposome hybrids. After surfactant removal, numerous liposomes (A, scale bar $=200 \mathrm{~nm}$ ) with associated nanoparticle clusters were observed. A close-up look $(B, C$, scale bar $=50 \mathrm{~nm})$ reveals the lipid bilayer splitting around the cluster. Subsequent tomographic reconstructions $(D$, scale bar $=50 \mathrm{~nm}$ ) additionally show the three-dimensional arrangements of the tightly packed nanoparticle clusters $(E)$.

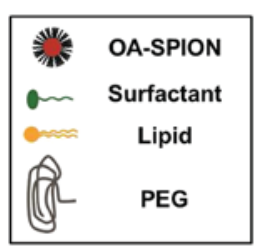

[OG] $<\mathrm{CMC}$

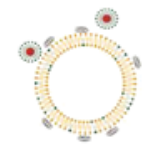

$[O G]=\mathrm{CMC}$


$[\mathrm{OG}] \gg \mathrm{CMC}$

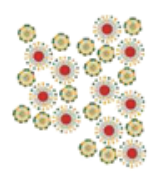

II.
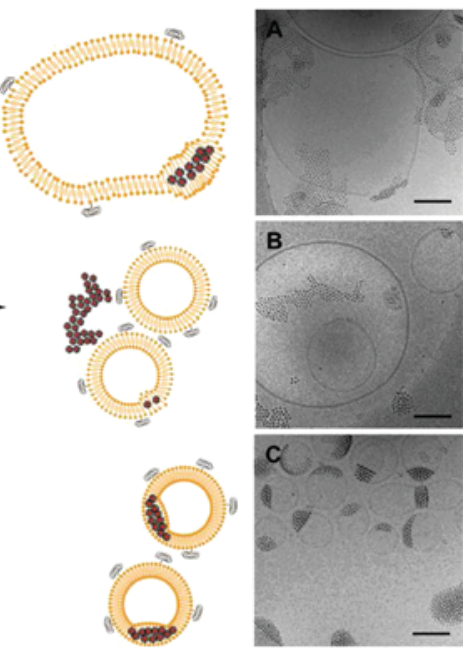

Figure 3. Initial surfactant concentration determines the incorporation efficiency. The surfactant concentration in relation to its critical micelle concentration has different effects on liposomes (I). When the surfactant is removed, different structures are obtained with varying initial concentrations (II). At sublytic initial concentrations (A), liposomes fuse together to form giant unilamellar vesicles, whereas most nanoparticles irreversibly aggregate. Concentrations around the CMC do not improve the yield (B). However, Janus magnetic liposomes (C) are obtained when the liposomes are entirely solubilized before surfactant dialysis. Scale bars $=100 \mathrm{~nm}$.

surfactant concentration closer to the CMC (Figure 3B) had no advantageous effects either. However, the incorporation efficiency was radically improved by initial superlytic surfactant concentrations ( $\gg C M C$, Figure $3 \mathrm{C}$ ) when both liposomes and nanoparticles had to entirely reassemble from independent micelles.

The major steps occurring during liposome reconstitution in the presence of SPIONs were captured by cryo-TEM (Figure 4). The observations can be interpreted with regards to existing models for vesicle formation upon surfactant depletion. ${ }^{32,43}$ However, the presence of foreign molecules (e.g., colloidal nanoparticles) significantly complicates the already complex phenomena encountered during the micelle-tovesicle transfer, as they necessarily interfere with all components. ${ }^{40}$ The liposome reconstitution procedure has been previously described as a three-step process including micellar equilibration (micellar growth by fusion or phospholipid exchange), ${ }^{44}$ bilayer closure (vesiculation), and vesicle growth. The observed 
A

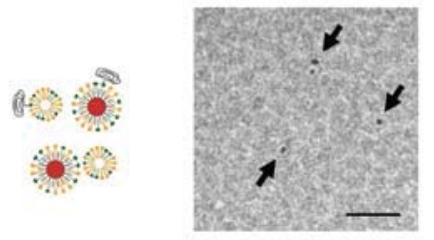

D
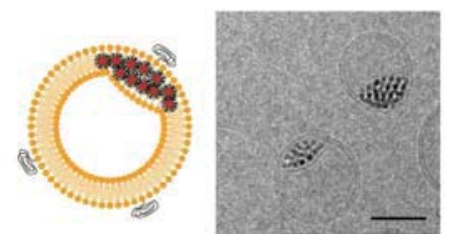

III. Post-vesiculation
B
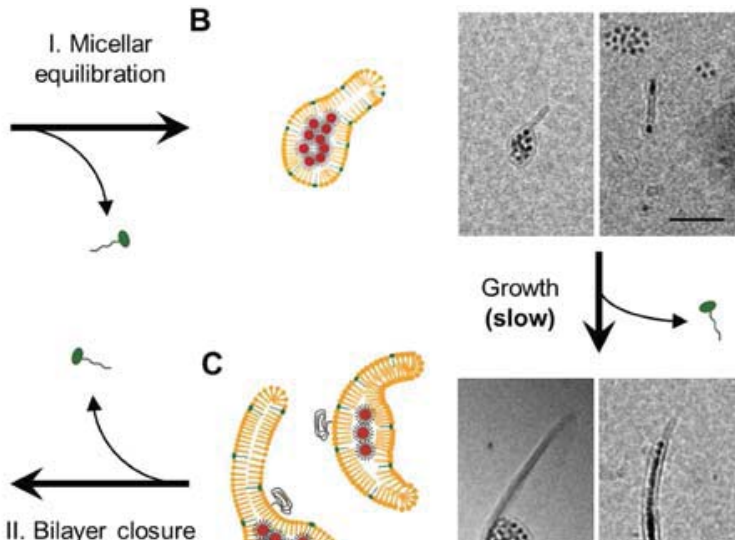

(fast)
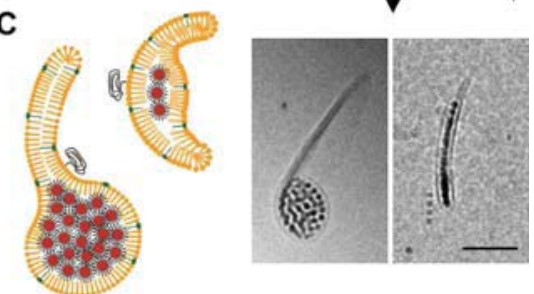

Figure 4. Micelles and nanoparticles undergo several intermediary assembly steps. At high surfactant concentrations, both nanoparticles and lipids remain in a stable micellar state (A). A thin phospholipid bilayer is clearly visible encircling the nanoparticles. When OG begins to be removed, the individual micelles start to merge and form, growing disk-like intermediate micelles $(B, C)$, preferably around already formed clusters. Once the disk has reached a critical size, the bilayer begins to bend and close. ${ }^{32,43}$ Postvesiculation follows upon total surfactant depletion and results in unilamellar Janus magnetic liposomes (D). Scale bars $=50 \mathrm{~nm}$.

continuity in formation (Figure 4, I and III) matches exactly with previous reports ${ }^{32,43}$ and further reveals how the liposomes grow around the clusters while slightly deforming them in the process.

These captions consequently explain the distinguishing Janus-like appearance and demonstrate how paramount it is that the nanoparticles first need to form flexible clusters before the liposomes begin to close around them. If this step does not occur, the single nanoparticles will remain in a stable micelle state, which generally coincides with previously documented observations. ${ }^{18,19}$ Interestingly, this approach can be applied to different nanoparticle sizes: In an additional trial, we observed that nanoparticles with a mean core diameter of $7.2 \pm 0.9 \mathrm{~nm}$ form similar structures, as well (Figure 5B), which shows that even nanoparticles up to $8.1 \mathrm{~nm}$ (Figure 5B, inset) can be inserted between the liposomal bilayer by following this methodology.

In contrast to the incorporation of flexible and adaptable proteins in the lipid membranes, the insertion of rigid nanoparticles is fundamentally different. The intrusion of hard spheres was reported to result in the conformational change of the lipid molecules themselves (e.g., stretching, compression, or tilting). ${ }^{22}$ In addition, the impact of the elastic deformation energy on the nanoparticle size has further been addressed on a theoretical basis. ${ }^{22}$ For hydrophobic quantum dots, it was consequently proposed that the incorporation of nanoparticles only smaller than $6.5 \mathrm{~nm}$ in the lipid bilayer over the nanoparticlemicelle complex is energetically favorable. ${ }^{22}$ However, our observations by cryo-TEM are contradictory, as the incorporated nanoparticle clusters are substantially larger and deform the lipid membrane in an
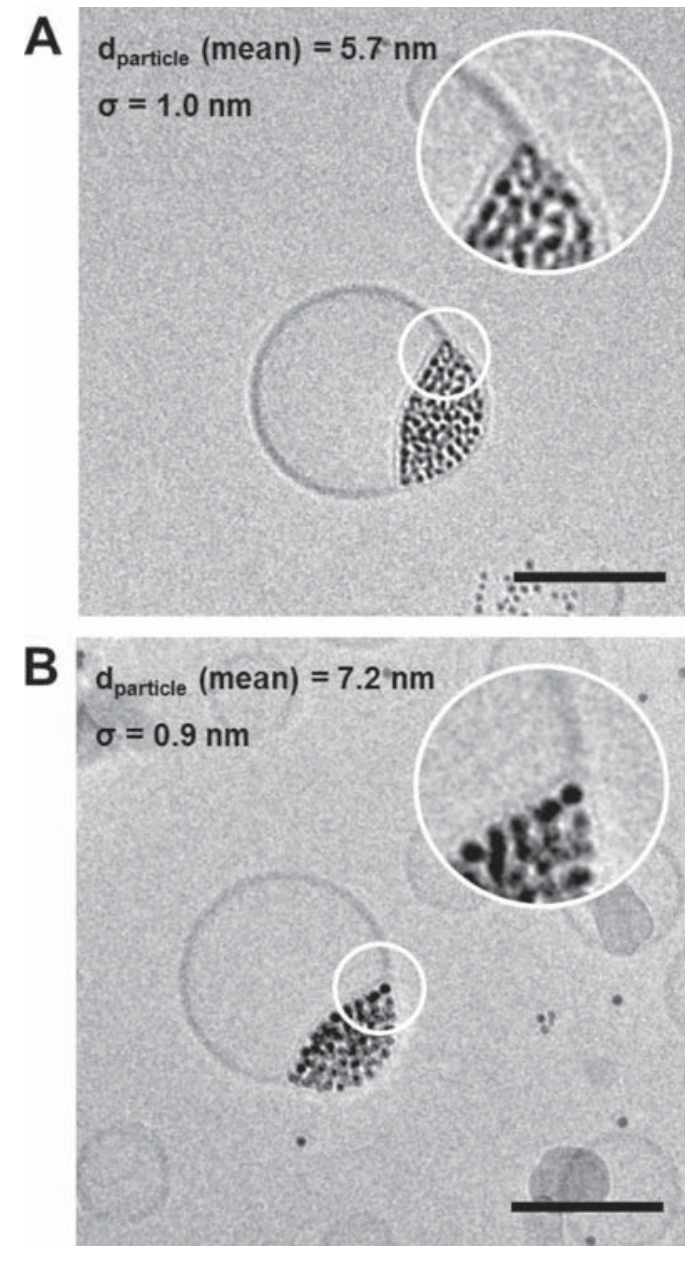

Figure 5. Cluster-liposome hybrids can be prepared with nanoparticles of different sizes. Vesicles similar to those obtained with relatively small nanoparticles (A) could be reproduced by using nanoparticles with a mean diameter superior to $6.5 \mathrm{~nm}$ (B). Scale bars = $100 \mathrm{~nm}$, insets = magnified by $2 \times$. 

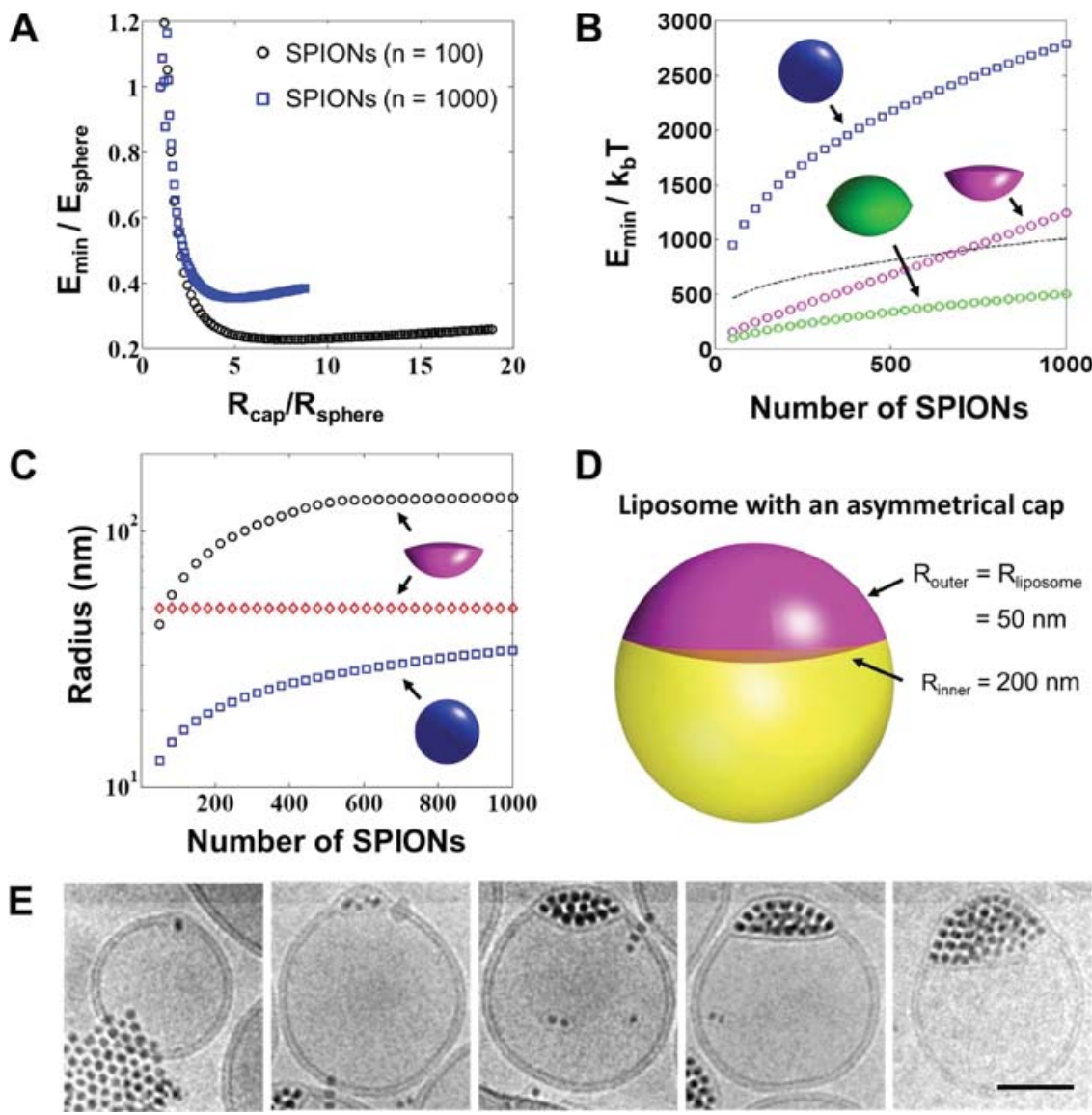

Figure 6. Energetics behind cluster-sized inclusions between a phospholipid membrane. (A) Inclusion energy of an inclusion with a double spherical cap geometry, as a function of the spherical cap radius for both 100 and 1000 nanoparticles. Both radius and energy are normalized by the corresponding values for a spherical inclusion. (B) Energy of an inclusion with an asymmetric spherical cap geometry, with one radius equal to the liposome radius (taken equal to $50 \mathrm{~nm}$ ) as a function of the number of nanoparticles in the inclusion. The energy of a corresponding spherical inclusion is additionally shown for comparison (blue), along with that of a spherical cluster covered by a lipid monolayer (black dashed line). (C) Radii of the asymmetric spherical cap inclusion as a function of the number of particles in the inclusion, as compared to the spherical inclusion radius. (D) Shape of a typical asymmetric inclusion with minimal energy. (E) Cryo-TEM images showing the membrane deformation with increasing number of embedded SPIONs. Scale bar $=50 \mathrm{~nm}$.

asymmetric fashion. Moreover, the outer lipid layer could recover its initial curvature by pushing the cluster toward the inner lipid leaflet. Although bilayers are extremely flexible with respect to their curvaturebending moment, ${ }^{39}$ the curvature change required to accommodate such large clusters is more important than previously indicated. The conclusions established previously ${ }^{22}$ were reached by comparing the energy required to deform a lipid bilayer with that required to cover a nanoparticle with a lipid monolayer. These finding are however only valid for rigid spherical geometries.

To explain why inclusions with a peculiar wedge shape are formed, we extended this model in order to accommodate inclusions with nonspherical shapes (Supporting Information). Since clusters composed of spheres are not fully rigid, it is conceivable that the distorted membrane would attempt to reduce the overall deformation energy by forcing the nanoparticles into clusters with lower curvature. To verify this hypothesis, the deformation energy for a flat membrane with two hexagonally packed inclusions was calculated (Figure 6A). The inclusions were designed as symmetric double spherical caps with volumes of either 100 or 1000 nanoparticles. As the spherical cap radius increases, accommodating such an inclusion requires a progressively lower curvature radius. Yet, because the inclusive volume remains identical, the inclusion occupies a progressively larger surface. Interestingly enough, a double spherical cap configuration leads to deformation energy values considerably lower than those of a corresponding spherical configuration (Figure 6B). The energy is even lower than that needed to cover a spherical cluster with a lipid monolayer. Nevertheless, an optimal size is reached because, as soon as the spherical cap radius increases above a certain value, the energy decrease caused by the reduced inclusion curvature radius does not compensate any longer for the increase in surface it occupies. This in turn leads to a further rise in energy and consequently clarifies why nonspherical inclusions are observed. 
To further inspect the peculiar asymmetric wedgeshaped inclusion, we repeated the calculations by supposing that the inclusion has the shape of an asymmetric double spherical cap, one of them having a fixed radius equal to that of the liposomes observed by cryo-TEM. The radius of the other spherical cap that minimizes the overall membrane deformation energy has been calculated under the constraint of a constant inclusion volume. The energy of such an optimal configuration was computed as a function of the number of nanoparticles within the inclusion (Figure 6B), together with the energy of the corresponding spherical inclusion. Once more, it is apparent that a spherical inclusion exhibits a much higher energy than an asymmetric double spherical cap, even though larger than that of a symmetric inclusion. The energy of an asymmetric inclusion is in any case smaller than that of a spherical cluster covered by a lipid monolayer (Figure 6B, dashed black line) until a nanoparticle count of approximately 700. Moreover, the energy of an asymmetric inclusion is at least by $1-2$ orders of magnitudes lower than that of the same number of individual nanoparticles covered by a lipid monolayer (Suppl. Figure 5). In Figure 6C, the radii of the two caps are shown, one being constant, the other much larger than the first one. This corresponds to an inclusion with a shape like the one illustrated in Figure 6D, which very closely resembles the inclusions observed by cryo-TEM (Figure 6E, right).

\section{DISCUSSION}

Clusters incorporated into the lipid bilayer stand out in comparison to other studies, where very small nanoparticles were observed to spread as monolayers within the liposome circumference. Many factors are determinant for these outcomes and the result of complex interplays between numerous simultaneously acting parameters. These include the physical membrane properties (i.e., bending energy, bending stiffness, lipid packing geometry, frustration, lateral tension, and interfacial energy), nanoparticle colloidal behavior, and finally the coinciding detergent effects on both of these systems. With regard to these effects, liposome reassembly by surfactant dialysis and the choice of surfactant have proven to be very suitable to control these parameters to an extent that favors the inclusion of structures of great inclusive volumes. Furthermore, the high-resolution cryogenic microscopy imaging techniques were essential for confirming the spatial location of these clusters and quantifying their inclusive size, thus underlining the importance of using the right methodology when characterizing vesicles. In terms of liposome membrane energetics, our fundamental considerations, backed by experimental validations, have shown that the present opportunities are consequently far broader than expected and show that not only higher quantities of nanoparticles can be incorporated between the thin bilayer but also larger structures and volumes.

\section{CONCLUSION}

When considering the future medical potential of this system, the higher nanoparticle density per vesicle provides a higher dose-response and subsequently more efficient products. By implementing clusters, size deficit for hyperthermia can be compensated by sheer number. Moreover, clustered SPIONs exhibit a significantly higher sensitivity in magnetic resonance imaging when compared to single nanoparticles, ${ }^{45-49}$ while still retaining their superparamagnetic properties. ${ }^{50}$ These circumstances make cluster-liposome hybrids particularly promising candidates when designing biocompatible and stimuli-sensitive nanocarriers.

Although such reflections are application- and material-dependent, these specifics show how radically the scope of effectiveness can be expanded by actuating this complex procedure in order to fully exploit the various opportunities at hand. To conclude, these findings offer new aspects on future developments of innovative wide-ranging nanocontainers for application-oriented purposes.

\section{METHODS}

Synthesis of Oleic-Acid-Coated Superparamagnetic Iron Oxide Nanoparticles. SPIONs stabilized by oleic acid were prepared by thermal decomposition. ${ }^{34}$ First, $2.2 \mathrm{~g}$ of iron(III) chloride hexahydrate ( $98 \%$, Sigma-Aldrich) and $7.5 \mathrm{~g}$ of sodium oleate $(97 \%$, TCl) were dissolved in $16 \mathrm{~mL}$ of pure ethanol (Sigma-Aldrich), $12 \mathrm{~mL}$ of Milli-Q water $(18.2 \mathrm{M} \Omega \cdot \mathrm{cm})$, and $28 \mathrm{~mL}$ of pure hexane (Sigma-Aldrich). The solution was heated to $70{ }^{\circ} \mathrm{C}$ and kept at this temperature for $4 \mathrm{~h}$. The black organic phase was washed three times with $10 \mathrm{~mL}$ of Milli-Q water and subsequently removed by rotary evaporation $\left(60^{\circ} \mathrm{C}, 520 \mathrm{mbar}\right)$.

Then, $7.9 \mathrm{~g}(8.8 \mathrm{mmol})$ of this iron oleate complex was dissolved in $52.6 \mathrm{~mL}$ of octyl ether (99\%, Sigma-Aldrich). Oleic acid ( $90 \%$, Sigma-Aldrich) was then added to the solution up to an iron oleate/oleic acid ratio of 2:1. The mixture was kept in a vacuum oven $\left(50{ }^{\circ} \mathrm{C}, 100 \mathrm{mbar}\right.$ ) for $2 \mathrm{~h}$ prior to the reaction and then heated according to the following ramp: $30-135^{\circ} \mathrm{C}=9.00^{\circ} \mathrm{C} / \mathrm{min}$ and $135-287^{\circ} \mathrm{C}=2.95^{\circ} \mathrm{C} / \mathrm{min}$. The suspension was kept at its refluxing temperature for $30 \mathrm{~min}$. Ethanol was added to precipitate the purified nanoparticles, which were then collected by centrifugation $(30000 \mathrm{~g}, 2 \mathrm{~min}$ ) and repeated three times.

Nanoparticle Phase Transfer with Surfactant. OA-SPIONs in hexane were mixed with Tris-acetate/ $\mathrm{NaCl}(40 \mathrm{mM} / 100 \mathrm{mM})$ buffer (Trizma actetate, sodium chloride, 99\%, Sigma-Aldrich) and $130 \mathrm{mM}$ of $\beta$-octyl glucoside. Hexane was then carefully removed at $50{ }^{\circ} \mathrm{C}$. To study the impact of surfactant concentration on colloidal stability, OG was removed by dialysis (Slide-ALyser, MWCO $=7 \mathrm{kDa}$, Thermo Scientific). Cryo-TEM imaging was performed within $24 \mathrm{~h}$.

Liposome Preparation. A phospholipid mixture with a transition phase temperature around $42^{\circ} \mathrm{C}$ was chosen with regards to future medical application. 
All phospholipids were purchased from Avanti Polar Lipids. 1,2-Dipalmitoyl-sn-glycero-3-phosphocholine (DPPC), 1,2-dimyristoyl-sn-glycero-3-phosphoethanolamine (DMPE), 1,2-dipalmitoyl-sn-glycero-3-phosphoethanolamine-PEG-2000 (DPPEPEG-2000), and cholesterol (99\%) were dispersed in chloroform. 1,2-Dipalmitoyl-sn-glycero-3-phospho-(1'-rac-glycerol) (sodium salt) (DPPG) was solubilized in a mixture of chloroform/ methanol (70:30). Liposomes were then prepared by lipid film rehydration. Cholesterol ( $10 \mathrm{wt} \%, 5.2 \mu \mathrm{mol})$, DPPC (43 wt \% $12.3 \mu \mathrm{mol})$, DPPG (24 wt $\%, 7.3 \mu \mathrm{mol})$, DMPE (19 wt $\%, 6.3 \mu \mathrm{mol})$, and DPPE-PEG-2000 (5 wt \%, $0.4 \mu \mathrm{mol}$ ) were then mixed in a round flask. The solvent was then removed by rotary evaporation to form a homogeneous lipid film. Tris-acetate/ $\mathrm{NaC}$ (40 mM/100 mM) was added to the lipid film and heated at $55{ }^{\circ} \mathrm{C}$ until the lipids were dissolved. The final lipid stock concentration was set to $10.5 \mathrm{mg} / \mathrm{mL}(15.8 \mathrm{mM})$. Unilamellar vesicles with a diameter of $100 \mathrm{~nm}$ were obtained by extrusion (mini-extruder, Avanti Polar Lipids).

Preparation of Janus Magnetic Liposomes. The liposome/ nanoparticle ratio was set to $1: 100$. Then, $500 \mu \mathrm{L}$ of PEGylated liposomes ( $5 \mathrm{mg}$ lipids $/ \mathrm{mL}$ ) and $456.5 \mu \mathrm{L}$ of surfactant-stabilized OA-SPIONs were mixed and briefly sonicated. The final volume was corrected to $1 \mathrm{~mL}$ with buffer and subjected to dialysis (Slide-A-Lyser, MWCO $=7 \mathrm{kDa}$, Thermo Scientific) against Trisacetate $/ \mathrm{NaCl}(40 \mathrm{mM} / 100 \mathrm{mM})$. The samples were then purified by size-exclusion chromatography (Sephadex G-25, PD-10). Large nanoparticle aggregates which formed during the process were removed by magnetic separation.

Cryo-TEM and Tomography: Sample Preparation and Analysis. Five microliters of the purified samples was pipetted on holey carbon-coated copper grids (400 mesh, Agar Scientific). The excess buffer was then carefully blotted away with filter paper (Whatman qualitative filter paper, grade 1). The grid was immediately plunge-frozen by using a commercial vitrification system (Vitrobot Mark IV, FEI). Following this, the grids were mounted in a Gatan 626 cryo-holder (Gatan Inc.) and investigated with a Tecnai F20 transmission electron microscope (FEI) at an operative voltage of $200 \mathrm{kV}$. Images were recorded with an Eagle camera (4096 × 4096 pixels, FEl) under low-dose conditions. The defocusing settings ranged between -1.5 and $-2.5 \mu \mathrm{m}$.

For cryo-electron tomography, images were acquired at sample holder tilting angles between $\pm 60^{\circ}$ with increments of $3^{\circ}$. The defocus was set to $-1.75 \mathrm{~nm}$. After reconstruction, 204 slices of $2 \mathrm{~nm}$ thickness each were obtained (Suppl. Figure 3).

Dynamic Light Scattering. Temporal correlation functions were recorded at three angles: $\theta=45,90$, and $120^{\circ}$ using a goniometer setup (LS Instruments) equipped with a $\mathrm{He}-\mathrm{Ne}$ laser $(\lambda=632.8 \mathrm{~nm})$ and a correlator (two-channel multiple tau correlator, min lag time $12.5 \mathrm{~ns}$, max lag time $50 \mathrm{~min}$ ). NMR tubes were used for measurements (Duran, $178 \times 4.95 \mathrm{~mm}, 300 \mathrm{MHz}$ ). Liposome suspensions were diluted with ultrafiltered buffer to have a final lipid concentration of $100 \mu \mathrm{g} / \mathrm{mL}$. Suspensions were measured three times at a temperature of $20^{\circ} \mathrm{C}$. The average radius was determined by second-order cumulant analysis. The CONTIN algorithm was used to quantify polydispersity.

Small-Angle X-ray Scattering (SAXS). SAXS measurements were performed in $2 \mathrm{~mm}$ quartz glass mark tubes (Hilgenberg \#4017520) on an S-Max 3000 (Rigaku Innovative Technologies). Sample and buffer were always measured using the same capillary for optimal background subtraction. OA-SPIONs in hexane $11 \mathrm{mg}$ iron $/ \mathrm{mL}$ ) and OG-OA-SPIONs were analyzed for $8 \mathrm{~h}$. OG-OA-SPIONs could not be analyzed after partial surfactant removal, as the clustered nanoparticles sedimented during measurement.

Data was analyzed using the SASfit software (http://kur.web. psi.ch/sans1/SANSSoft/sasfit.html, as of February 2014). For OA-SPIONs, the best curve fitting was obtained using a sphere model (Supporting Information). The distribution of the particles follows a log-normal distribution.

Model Calculations. The presented mathematical model is an extension of the model established by Wi et al. ${ }^{22}$ Briefly, the model computes the inclusion energy as the sum of both bending and stretching energy. The bending energy depends on the difference between the natural curvature of the lipid bilayer and the one induced by an inclusion. The stretching contribution relies on the stretching extent of the lipid monolayer from its unperturbed thickness. The model further assumes that the lipid monolayer profile can be divided into two regions: the first one (I) with a curvature equal to the one of the inclusion, and a second one (II) with a constant curvature that can be derived from purely geometric arguments (Suppl. Figure 7). The point where region I passes over to region II is determined by a minimization of the overall energy. In contrast to the previously developed model, ${ }^{22}$ this model is predicated on a spherical inclusion but assumes that inclusions can be described by spherical caps. The cap shape has been chosen so as to retain almost the same mathematical approach, but with a degree of freedom given by the choice of the position of the sphere center to which the cap belongs.

The overall energy change in the lipid monolayer due to the presence of the inclusion is given by

$\Delta E=\Delta E_{\text {stretch }}+\Delta E_{\text {bend }}$

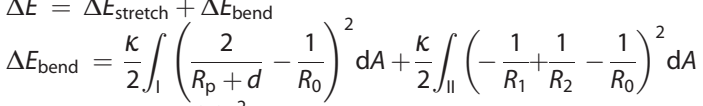

$\Delta E_{\text {stretch }}=\frac{K}{2} \int_{\|}\left(\frac{u}{d}\right)^{2} \mathrm{~d} A$

where $d$ is the thickness of the lipid monolayer, $R_{\mathrm{p}}$ the radius of the inclusion (i.e., of the spherical cap), $R_{1}$ and $R_{2}$ are the two curvature radii of the inclusion in region II, $R_{0}$ is the spontaneous curvature radius of the lipid monolayer, $u$ the stretching of the monolayer, $K$ the stretching modulus, and $\kappa$ the bending modulus. The values of $\mathrm{d}, R_{0}, K$, and $\kappa$ are the same as used previously. ${ }^{22}$ A sensitivity analysis has been run to test the results in response to changes in these values, which are displayed in Suppl. Figure 6. In order to compute the integrals in eq 1 , it is necessary to determine $u, R_{1}$, and $R_{2}$.

Referring to Suppl. Figure 7, we have

$$
\left\{\begin{array}{l}
R_{1}+d+c=b \\
\left(R_{1}+d+R_{\mathrm{p}}\right) \cos (\theta)=b+c \\
\left(R_{1}+d+R_{\mathrm{p}}\right) \sin (\theta)=a
\end{array}\right.
$$

$$
\frac{R_{1}}{R_{2}}=\frac{a-r}{r}
$$

where $u$ is instead obtained in different ways, depending on whether we are referring to region $\|$ or $\|_{b}$. In region $\|_{b}$, we have

$$
\left\{\begin{array}{l}
u(r)+R_{1}+d=\frac{b}{\cos (\varphi)}=\frac{b}{\sqrt{1-\sin ^{2}(\varphi)}} \\
\sin (\varphi)=\frac{a-r}{R_{1}}
\end{array}\right.
$$

On the other hand, in region $\mathrm{Il}_{\mathrm{a}}$, we have instead

$$
\left\{\begin{array}{l}
u(r)+R_{1}+d+\sqrt{y_{c}^{2}+\left(x_{c}-x_{r}\right)}=\frac{b}{\cos (\varphi)}=\frac{b}{\sqrt{1-\sin ^{2}(\varphi)}} \\
\sin (\varphi)=\frac{a-r}{R_{1}}
\end{array}\right.
$$

where

$$
\left\{\begin{array}{l}
y_{\mathrm{c}}=b+\left(\frac{\sqrt{R_{1}^{2}-(a-r)^{2}}}{a-r}\right)\left(x_{\mathrm{c}}-a\right) \\
x_{\mathrm{c}}^{2}+\left(y_{\mathrm{c}}+c\right)^{2}=R_{\mathrm{p}}^{2} \\
x_{\mathrm{r}}=a-b\left(\frac{a-r}{\sqrt{R_{1}^{2}-(a-r)^{2}}}\right)
\end{array}\right.
$$

We have two additional degrees of freedom with respect to the original model: the position of the inclusion center $c$ and the inclusion radius; $c$ is determined by imposing that conservation of (half of) the inclusion volume:

$$
V=\frac{\pi}{3}\left(R_{\mathrm{p}}-c\right)^{2}\left(2 R_{\mathrm{p}}+c\right)
$$


Note that the inclusion volume can be independently computed from the number and volume of each nanoparticle assuming that they assemble into a hexagonal packing. The inclusion radius and the value of angle $\theta$ are obtained by minimizing the overall energy. In order to mimic more closely what happens in the case of a liposome, the following assumption has been made: since the results show that the cap radius tends to be large, it is expected that one of the inclusion radi will be equal to the one of the liposome, while the other one will have to be determined by energy minimization. The following additional geometrical constraints thus need to be applied:

$$
\left\{\begin{array}{l}
V=\frac{\pi}{3}\left(R_{\mathrm{p}, 1}-c_{1}\right)^{2}\left(2 R_{\mathrm{p}, 1}+c_{1}\right)+\frac{\pi}{3}\left(R_{\mathrm{p}, 2}-c_{2}\right)^{2}\left(2 R_{\mathrm{p}, 2}+c_{2}\right) \\
R_{\mathrm{p}, 1}^{2}-c_{1}^{2}=R_{\mathrm{p}, 2}^{2}-c_{2}^{2}
\end{array}\right.
$$

The first equation denotes the volume conservation. The second one expresses the geometrical requirement that the radius of the base of the caps has to be the same for both caps which share the same base. Numerically, this has been reduced to a single nonlinear algebraic equation.

Conflict of Interest: The authors declare no competing financial interest.

Acknowledgment. The authors would like to thank K. van Gruijthuijsen for her help with the scattering experiments and the fitting using SASfit. Moreover, they would like to thank S. Balog for his advice and help with scattering experiments. The support of the Dr. Alfred Bretscher Fund is gratefully acknowledged, and access to cryo-TEM was kindly provided by the Microscopy Imaging Centre of the University of Bern. This work was supported by the Swiss National Science Foundation (126104, PP00P2-123373/1, and PP00P2133597/1), the Adolphe Merkle Foundation, and the University of Fribourg.

Supporting Information Available: Additional information on detailed procedures and characterization, along with an animated tomographic rendering is available. This material is available free of charge via the Internet at http://pubs.acs.org.

\section{REFERENCES AND NOTES}

1. Immordino, M. L.; Dosio, F.; Cattel, L. Stealth Liposomes: Review of the Basic Science, Rationale, and Clinical Applications, Existing and Potential. Int. J. Nanomed. 2006, 1, 297-315.

2. Torchilin, V. P. Recent Advances with Liposomes as Pharmaceutical Carriers. Nat. Rev. Drug Discovery 2005 4, 145-160.

3. Yatvin, M. B.; Weinstein, J. N.; Dennis, W. H.; Blumenthal, R. Design of Liposomes for Enhanced Local Release of Drugs by Hyperthermia. Science 1978, 202, 1290-1293.

4. Kono, K.; Igawa, T.; Takagishi, T. Cytoplasmic Delivery of Calcein Mediated by Liposomes Modified with a $\mathrm{pH}$ Sensitive Poly(ethylene glycol) Derivative. Biochim. Biophys. Acta 1997, 1325, 143-154.

5. Hafez, I. M.; Ansell, S.; Cullis, P. R. Tunable pH-Sensitive Liposomes Composed of Mixtures of Cationic and Anionic Lipids. Biophys. J. 2000, 79, 1438-1446.

6. Gerasimov, O. V.; Boomer, J. A.; Qualls, M. M.; Thompson, D. H. Cytosolic Drug Delivery Using $\mathrm{pH}$ - and LightSensitive Liposomes. Adv. Drug Delivery Rev. 1999, 38, 317-338.

7. Bibi, S.; Lattmann, E.; Mohammed, A. R.; Perrie, Y. Trigger Release Liposome Systems: Local and Remote Controlled Delivery? J. Microencapsulation 2012, 29, 262-276.

8. Al-Jamal, W. T.; Kostarelos, K. Liposomes: From a Clinically Established Drug Delivery System to a Nanoparticle Platform for Theranostic Nanomedicine. Acc. Chem. Res. 2011, 44, 1094-1104.

9. Deserno, M.; Gelbart, W. M. Adhesion and Wrapping in Colloid-Vesicle Complexes. J. Phys. Chem. B 2002, 106, 5543-5552.

10. Nel, A.; Xia, T.; Mädler, L.; Li, N. Toxic Potential of Materials at the Nanolevel. Science 2006, 311, 622-627.
11. Leroueil, P. R.; Hong, S.; Mecke, A.; Baker, J. R.; Orr, B. G.; Banaszak Holl, M. M. Nanoparticle Interaction with Biological Membranes: Does Nanotechnology Present a Janus Face. Acc. Chem. Res. 2007, 40, 335-342.

12. Roiter, Y.; Ornatska, M.; Rammohan, A. R.; Balakrishnan, J.; Heine, D. R.; Minko, S. Interaction of Nanoparticles with Lipid Membrane. Nano Lett. 2008, 8, 941-944.

13. Ginzburg, V.; Balijepalli, S. Modeling the Thermodynamics of the Interaction of Nanoparticles with Cell Membranes. Nano Lett. 2007, 7, 3716-3722.

14. Alexeev, A.; Uspal, W. E.; Balazs, A. C. Harnessing Janus Nanoparticles To Create Controllable Pores in Membranes. ACS Nano 2008, 2, 1117-1122.

15. Mornet, S.; Lambert, O.; Duguet, E.; Brisson, A. The Formation of Supported Lipid Bilayers on Silica Nanoparticles Revealed by Cryoelectron Microscopy. Nano Lett. 2005, 5, 281-285.

16. Lee, H.; Larson, R. G. Lipid Bilayer Curvature and Pore Formation Induced by Charged Linear Polymers and Dendrimers: The Effect of Molecular Shape. J. Phys. Chem. B 2008, 112, 12279-12285.

17. Chen, Y.; Bose, A.; Bothun, G. D. Controlled Release from Bilayer-Decorated Magnetoliposomes via Electromagnetic Heating. ACS Nano 2010, 4, 3215-3221.

18. Amstad, E.; Kohlbrecher, J.; Müller, E.; Schweizer, T.; Textor, M.; Reimhult, E. Triggered Release from Liposomes through Magnetic Actuation of Iron Oxide Nanoparticle Containing Membranes. Nano Lett. 2011, 11, 16641670.

19. Gopalakrishnan, G.; Danelon, C.; Izewska, P.; Prummer, M.; Bolinger, P.-Y.; Geissbühler, I.; Demurtas, D.; Dubochet, J.; Vogel, H. Multifunctional Lipid/Quantum Dot Hybrid Nanocontainers for Controlled Targeting of Live Cells. Angew. Chem., Int. Ed. 2006, 45, 5478-5483.

20. Rasch, M. R.; Rossinyol, E.; Hueso, J. L.; Goodfellow, B. W.; Arbiol, J.; Korgel, B. A. Hydrophobic Gold Nanoparticle SelfAssembly with Phosphatidylcholine Lipid: MembraneLoaded and Janus Vesicles. Nano Lett. 2010, 10, 37333739.

21. Binder, W. H.; Sachsenhofer, R.; Farnik, D.; Blaas, D. Guiding the Location of Nanoparticles into Vesicular Structures: A Morphological Study. Phys. Chem. Chem. Phys. 2007, 9, 6435-6441.

22. Wi, H. S.; Lee, K.; Pak, H. K. Interfacial Energy Consideration in the Organization of a Quantum Dot-Lipid Mixed System. J. Phys.: Condens. Matter 2008, 20, 494211.

23. Preiss, M. R.; Bothun, G. D. Stimuli-Responsive LiposomeNanoparticle Assemblies. Expert Opin. Drug Delivery 2011, 8, 1025-1040.

24. Kumar, C. S. S. R.; Mohammad, F. Magnetic Nanomaterials for Hyperthermia-Based Therapy and Controlled Drug Delivery. Adv. Drug Delivery Rev. 2011, 63, 789-808.

25. Mehdaoui, B.; Meffre, A.; Carrey, J.; Lachaize, S.; Lacroix L.-M.; Gougeon, M.; Chaudret, B.; Respaud, M. Optimal Size of Nanoparticles for Magnetic Hyperthermia: A Combined Theoretical and Experimental Study. Adv. Funct. Mater. 2011, 21, 4573-4581.

26. Thiesen, B. Jordan, A. Clinical Applications of Magnetic Nanoparticles for Hyperthermia. Int. J. Hyperthermia 2008, 24, 467-474.

27. Nappini, S.; Bombelli, F. B.; Bonini, M.; Nordèn, B.; Baglioni, P. Magnetoliposomes for Controlled Drug Release in the Presence of Low-Frequency Magnetic Field. Soft Matter 2009, 6, 154.

28. Tai, L.-A.; Tsai, P.-J.; Wang, Y.-C.; Wang, Y.-J.; Lo, L.-W.; Yang, C.-S. Thermosensitive Liposomes Entrapping Iron Oxide Nanoparticles for Controllable Drug Release. Nanotechnology 2009, 20, 135101.

29. Pankhurst, Q. A.; Connolly, J.; Jones, S. K.; Dobson, J. Applications of Magnetic Nanoparticles in Biomedicine. J. Phys. D: Appl. Phys. 2003, 36, R167-R181.

30. Qin, J.; Laurent, S.; Jo, Y. S.; Roch, A.; Mikhaylova, M.; Bhujwalla, Z. M.; Muller, R. N.; Muhammed, M. A HighPerformance Magnetic Resonance Imaging T2 Contrast Agent. Adv. Mater. 2007, 19, 1874-1878. 
31. Laurent, S.; Forge, D.; Port, M.; Roch, A.; Robic, C.; Vander Elst, L.; Muller, R. N. Magnetic Iron Oxide Nanoparticles: Synthesis, Stabilization, Vectorization, Physicochemical Characterizations, and Biological Applications. Chem. Rev. 2008, 108, 2064-2110.

32. Lasic, D. D. The Mechanism of Vesicle Formation. Biophys. J. 1988, 256, 1-11.

33. Mehta, S: Somasundaran, P: Yu, X: Krishnakumar, S Colloid Systems and Interfaces Stability of Dispersions through Polymer and Surfactant Adsorption. In Handbook of Surface and Colloid Chemistry; Birdi, K., Ed.; CRC Press: Boca Raton, FL, 2008; pp 155-196.

34. Park, J.; An, K.; Hwang, Y.; Park, J.-G.; Noh, H.-J.; Kim, J.-Y. Park, J.-H.; Hwang, N.-M.; Hyeon, T. Ultra-Large-Scale Syntheses of Monodisperse Nanocrystals. Nat. Mater. 2004, 3, 891-895.

35. Bogusz, S.; Venable, R. M.; Pastor, R. W. Molecular Dynamics Simulations of Octyl Glucoside Micelles: Structural Properties. J. Phys. Chem. B 2000, 104, 5462-5470.

36. Paternostre, M. T.; Roux, M.; Rigaud, J. L. Mechanisms of Membrane Protein Insertion into Liposomes During Reconstitution Procedures Involving the Use of Detergents. 1. Solubilization of Large Unilamellar Liposomes (Prepared by Reverse-Phase Evaporation) by Triton X-100, Octyl Glucoside, and Sodium Cholate. Biochemistry 1988, 27, 2668-2677.

37. Mimms, L. T.; Zampighi, G.; Nozaki, Y.; Tanford, C.; Reynolds, J. A. Phospholipid Vesicle Formation and Transmembrane Protein Incorporation Using Octyl Glucoside. Biochemistry 1981, 20, 833-840.

38. Shinoda, K.; Yamanaka, T.; Kinoshita, K. Surface Chemical Properties in Aqueous Solutions of Non-ionic Surfactants Octyl Glycol Ether, $\alpha$-Octyl Glyceryl Ether and Octyl Glucoside. J. Phys. Chem. 1959, 63, 648-650.

39. Sackmann, E. Physical Basis of Self-Organization and Function of Membranes: Physics of Vesicles. In Handbook of Biological Physics; Elsevier: Amsterdam, 1995; pp 213-304.

40. Ollivon, M.; Eidelman, O.; Blumenthal, R.; Walter, A. MicelleVesicle Transition of Egg Phosphatidylcholine and Octylglucoside. Biochemistry 1988, 27, 1695-1703.

41. Ménager, C.; Guemghar, D.; Perzynski, R.; Lesieur, S. Cabuil, V. Lipid Bilayer Elasticity Measurements in Giant Liposomes in Contact with a Solubilizing Surfactant. Langmuir 2008, 24, 4968-4974.

42. Bonnaud, C.; Vanhecke, D.; Demurtas, D.; Rothen-Rutishauser B.; Petri-Fink, A. Spatial SPION Localization in Liposome Membranes. IEEE Trans. Magn. 2013, 49, 166-171.

43. Leng, J.; Egelhaaf, S. U.; Cates, M. E. Kinetics of the Micelleto-Vesicle Transition: Aqueous Lecithin-Bile Salt Mixtures. Biophys. J. 2003, 85, 1624-1646.

44. Levy, D.; Gulik, A.; Seigneuret, M.; Rigaud, J.-L. Phospholipid Vesicle Solubilization and Reconstitution by Detergents. Symmetrical Analysis of the Two Processes Using Octaethylene Glycol Mono-n-dodecyl Ether. Biochemistry 1990, 29, 9480-9488.

45. Hayashi, K. Superparamagnetic Nanoparticle Clusters for Cancer Theranostics Combining Magnetic Resonance Imaging and Hyperthermia Treatment. Theranostics 2013, 3, 366-376.

46. Paquet, C: Haan, H. W: Leek, D. M. : Lin, H.-Y:Xiang, B:Tian G.; Kell, A.; Simard, B. Nanobeads Highly Loaded with Superparamagnetic Nanoparticles Prepared by Emulsification and Seeded-Emulsion Polymerization. ACS Nano 2011, 5, 3104-3112

47. Xie, X.; Zhang, C. Controllable Assembly of Hydrophobic Superparamagnetic Iron Oxide Nanoparticle with mPEGPLA Copolymer and Its Effect on MR Transverse Relaxation Rate. J. Nanomater. 2011, 2011, 1-7.

48. Berret, J.-F.; Schonbeck, N.; Gazeau, F.; El Kharrat, D. Sandre, O.; Vacher, A.; Airiau, M. Controlled Clustering of Superparamagnetic Nanoparticles Using Block Copolymers: Design of New Contrast Agents for Magnetic Resonance Imaging. J. Am. Chem. Soc. 2006, 128, 1755 1761.
49. Paquet, C.; Pagé, L.; Kell, A.; Simard, B. Nanobeads Highly Loaded with Superparamagnetic Nanoparticles Prepared by Emulsification and Seeded-Emulsion Polymerization. Langmuir 2010, 26, 5388-5396.

50. Qiu, P.; Jensen, C.; Charity, N.; Towner, R.; Mao, C. Oil Phase Evaporation-Induced Self-Assembly of Hydrophobic Nanoparticles into Spherical Clusters with Controlled Surface Chemistry in an Oil-in-Water Dispersion and Comparison of Behaviors of Individual and Clustered Iron Oxide Nanoparticles. J. Am. Chem. Soc. 2010, 132, 17724-17732. 